\title{
The Strategic Value of Quantity Forcing Contracts*
}

\author{
David Martimort ${ }^{\dagger} \quad$ Salvatore Piccolo ${ }^{\ddagger}$
}

2nd February 2009

\begin{abstract}
We explore the strategic value of quantity forcing contracts in a manufacturerretailer environment under both adverse selection and moral hazard. Manufacturers dealing with (exclusive) competing retailers may prefer to leave contracts silent on retail prices, whenever other aspects of the retailers' activity remain nonverifiable. Two effects are at play when moving from retail price maintenance to quantity forcing. First, restricting screening possibilities may increase retailers' rent. Second, such restriction affects downstream competition. This latter effect may justify using quantity forcing contracts and, more generally, shed light on a novel source of contractual incompleteness.
\end{abstract}

JEL: D2, D23, D82, K21.

Keywords: Asymmetric Information, Competing Hierarchies, Incomplete Contracts, Quantity Forcing, Resale Price Maintenance, Vertical Contracting.

${ }^{*}$ We are grateful to Sandeep Baliga, Pierpaolo Battigalli, Jim Dana, Chiara Fumagalli, Matteo Galizzi, Jakub Kastl, Massimo Marrelli, Eric Maskin, Alessandro Pavan, Jean-Charles Rochet, Bill Rogerson, Jean Tirole, Mike Whinston, two referees and the Editor Andy Postlewaite for useful remarks and suggestions. We also thank seminar participants at Bruxelles DG Competition, Brescia, CSEF-IGIER Symposium in Economics and Institutions (Anacapri, 2006), Duke, Luiss (Rome), Northwestern, Napoli, Padova, Salerno and Toulouse. A previous version of this paper has circulated under the title "The Strategic Value of Incomplete Contracts for Competing Hierarchies". Errors are ours.

${ }^{\dagger}$ Toulouse School of Economics and EHESS, France, martimor@cict.fr

‡University of Napoli Federico II, CSEF and Toulouse School of Economics, spiccolo@unisa.it 


\section{Introduction}

Common wisdom suggests that optimal contracts should limit as much as possible agents' discretion within agency relationships. Principals should then profitably exploit all available screening and monitoring instruments in order to prevent agents' misbehavior. Nevertheless, contractual rules seldom display such high degree of complexity. In practice, contracts appear rather simple and, more strikingly, quite often fail to specify verifiable obligations of the parties.

Arm's length relationships are widespread business practices. An archetypal example, which we study in this paper, is provided by manufacturer-retailer relationships. Particular emphasis has indeed been put forward by the recent IO literature on the very incomplete nature of contracts regulating trade between vertically related firms: ${ }^{1}$ not only manufacturers often delegate marketing activities to retailers, but they also frequently give up vertical control, by refusing to impose contractual restraints that would reduce agency costs and, in turn, potentially improve upon allocative efficiency. ${ }^{2}$

What is the source of contract incompleteness in these markets? Taking a broader perspective, existing agency models have provided few rationales for why contracts are incomplete. One line of thoughts, for instance, has appealed to unforeseen contingencies, and costs of writing and enforcing contracts (see Jean Tirole, 1999). But this body of work is usually silent on the strategic value that competition may confer to arrangements that seem too simple or incomplete.

Our paper has two main goals. First, we shed new light on the benefits for manufacturers to forego the contracting ability on retail prices and prefer simpler (less complete) quantity forcing contracts. We offer a new rationale for the widespread use of simple contracts in vertical relationships and account for a hitherto neglected link between downstream externalities and contractual design in such markets. Second, we derive implications of our analysis for the wider theoretical debate over contract incompleteness, and show how our results complement existing models.

We frame the analysis in a manufacturer-retailer context, which allows for both adverse selection and moral hazard. In this specific setting our objective is to investigate the extent to which simple quantity forcing contracts can be strategically used by manufacturers and preferred to more complete arrangements based on retail price control. Moving from the standard sequential monopolies framework to games where the retail market is 'imperfectly competitive', the choice of less complete contracts, like quantity forcing arrangements, results from the interplay between the agency costs associated to alternative incentive schemes and the externalities that competing retailers exert on each other. We show that when some aspects of the retailers' downstream activities are nonverifiable, foregoing the opportunity to contract on retail prices might have a strategic value.

\footnotetext{
${ }^{1}$ The applied literature on this topic (Francine Lafontaine and Margaret Slade, 1997, among many others) has recently argued that the empirical evidence, fairly consistent across industries and firms, quite often appears to be inconsistent with some aspects of the theoretical predictions based on agency theory.

${ }^{2}$ There are many other examples of incomplete contracts. Managerial contracts are typically vague or silent on the competitive and organizational objectives that managers should pursue; lenders usually leave entrepreneurs free to perform certain tasks that affect the profitability of their ventures; insurance companies monitor only to a limited extent the behavior of insurees, to name only a few.
} 
To make this point as clear as possible for our purpose, consider a one-sided hierarchy model where two downstream retailers compete by setting quantities, and assume that while one of these (hereafter the agent) deals with an exclusive upstream supplier (the principal) to acquire an essential input for production, his competitor is vertically integrated and has access to the input at no cost. ${ }^{3}$ Demands are uncertain and only downstream firms observe a payoff relevant signal before contracts are designed: an adverse selection problem. Moreover, the non-integrated agent exerts a nonverifiable demandenhancing activity (effort) which may also affect the competitor's demand: a moral hazard issue. ${ }^{4}$ The manufacturer hires the retailer before production occurs, but after uncertainty has realized, and he has all bargaining power in dictating the terms of trade.

Two alternative contractual regimes are compared: the principal can either commit to a simple quantity forcing scheme $(\mathrm{QF})$, or to a more sophisticated arrangement, comparable to resale price maintenance (RPM). These arrangements differ in their degree of contractual completeness. Specifically, QF is more incomplete than RPM in the sense that, beyond fixing the quantity supplied to final consumers, it leaves the downstream agent free to choose its most preferred level of promotional effort. Instead, a RPM mechanism also restrains the retail price charged to final consumers in addition to fixing the quantity supplied in the final market.

Two contrasting effects are at play once one moves from RPM to QF. On the one hand, QF leaves more possibilities for the agent to enjoy an information rent because with such a contract he appropriates a greater share of the return on improving own demand. On the other hand, foregoing price control might have a strategic value for the principal. By allowing his agent to respond more efficiently to competition, it can induce a favorable behavior by the competing retailer at the market stage. Yet, while the former effect has already been discussed in previous work, ${ }^{5}$ the second is novel.

The analysis provides simple conditions under which QF contracting may be preferred when retailers impose either positive or negative externalities on each other. To understand this point, it is worthwhile observing that when downstream demands are independent, that is retailers have monopolistic power in their own markets, RPM is clearly preferred by the principal. Indeed, complete contracting provides more tools to better extract the retailer's private information on demand, whereby allowing to reach a better trade-off between efficiency and rent extraction within this principal-agent pair. Competition between retailers, though, brings a novel channel through which foregoing retail price affects the supplier's profit. By committing to leave his downstream agent free to set some aspects of his performance, a manufacturer can influence in her own interest the subsequent retail game.

The key feature of our environment is the link between market and non-market (ef-

\footnotetext{
${ }^{3}$ Allowing for a more symmetric framework with two competing hierarchies does not alter the main insights of the one-sided hierarchy model that we analyze here (see Jakub Kastl, David Martimort and Salvatore Piccolo, 2008).

${ }^{4}$ Distributors can indeed provide a wide range of services that affect demand. Free delivery, pre-sales advices to potential buyers, show rooms, and after-sales services play a key role in enhancing demand. Looking at supermarket data relative to the Chicago area, Judith A. Chevalier, Anil K. Kashyap and Peter E. Rossi (2003) document the importance of retailers' activity in price determination and the role of the retailers' advertising as a way of competing for customers.

${ }^{5}$ See Benjamin F. Blair and Tracy R. Lewis (1994), Esther Gal-Or (1991b) and Martimort and Piccolo (2007) for instance.
} 
fort) externalities that downstream retailers impose on each other. We show that when both kinds of externalities are negative (resp. positive), that is goods are substitutes (resp. complements) and the retailer's effort has a selfish (resp. cooperative) nature, the principal strictly prefers a QF contract instead of the more complete RPM, provided that information costs are not very large. Essentially, by inducing more effort on the agent's side, foregoing the ability to contract on prices shifts around the competitors' equilibrium output supply. This has a positive strategic effect as long as market and non-market externalities have the same sign. By contrast, in a free-riding environment, which emerges when goods are substitutes and efforts have a cooperative value, RPM is the best choice. In this case, increasing the effort of the agent through the contractual channel is not optimal as it makes downstream competitors more aggressive via the positive non-market externality.

This result is fairly general and, although we have developed the formal arguments in a stylized IO example, our conclusions are of wider scope. They can rationalize the use of simple contracts basically within any competing hierarchies model involving vertical and horizontal contractual externalities, be it procurement contracting, executive compensations, patent licensing, insurance or credit relationships, to name only a few.

The paper is structured as follows. Section 2 lays out the model. Section 3 provides the equilibrium characterization. Section 4 analyzes the strategic value of QF contracts. Section 5 offers an overview of the related IO literature and comments the link between our paper and the body of work on incomplete contracting. Section 6 concludes.

\section{The model}

Players and environment: Consider a simple retail industry where two downstream outlets, $R_{1}$ and $R_{2}$, producing symmetrically differentiated products compete by setting quantities. The production of each of these final outputs requires an essential raw input which is supplied at the upstream level. To make the analysis as sharp as possible for our purpose, consider a one-sided hierarchy model and assume that, while $R_{1}$ must deal with an independent supplier $S_{1}$ to secure the input, $R_{2}$ is vertically integrated with his own supplier $S_{2}$ and produces as a unique entity, hereafter labeled $S_{2}-R_{2}$. As we shall see, this simple structure already brings out clearly the relevant trade-offs that $S_{1}$ faces when choosing between QF and RPM.

The system of (inverse) market demands is defined by:

$$
p_{1}\left(\theta, e_{1}, q_{1}, q_{2}\right)=\theta+e_{1}-q_{1}+\rho q_{2}, \quad \text { and } \quad p_{2}\left(\theta, e_{1}, q_{2}, q_{1}\right)=\theta+\sigma e_{1}-q_{2}+\rho q_{1} .
$$

where $q_{i}$ is the quantity produced of good $i, p_{i}$ is the retail price charged for this product, and $\theta$ is a common shock affecting both demands. The demand parameter $\theta$ is uniformly distributed on the compact support $\Theta=[\underline{\theta}, \bar{\theta}]$ (we denote $\Delta \theta=\bar{\theta}-\underline{\theta}>0$ and assume that $\underline{\theta}$ is large enough to maintain positive output and effort in all circumstances below). The realization of $\theta$ is private information of retailers at the time contracts are signed, and $e_{1}$ denotes a non-observable demand-enhancing activity (effort) performed by retailer $R_{1}{ }^{6}$

\footnotetext{
${ }^{6}$ Neither $S_{1}$ nor $S_{2}-A_{2}$ can observe $e_{1}$.
} 
The effort variable is meant to capture any kind of non-market activity performed by $R_{1}$, such as production of indivisible services, investment in advertising or pre-sale advises to potential buyers, that is not directly controlled by $S_{1} \cdot{ }^{7}$ Such kind of effort has two effects on the demand system. Clearly, it enhances own consumers' willingness to pay, but it may also influence the competitor's demand. This assumption seems reasonable in at least two cases. First, when effort is interpreted as production of indivisible services bundled with the final product, it might have a negative impact on competitors' demand if goods are substitutes; and the opposite obtains for complements. Second, when effort captures pre-sale services or generic advertising, it could well be the case that information on the product's existence benefits also competitors: a free-riding story (e.g., Mathewson and Winter, 1984).

Following Yeon-Koo Che and Donald B. Hausch (1999), we distinguish these alternative scenarios depending on the effort value, which is cooperative for $\sigma>0$, and selfish otherwise. Throughout we shall assume that $|\sigma| \leq 1$ in order to guarantee that owneffort effects are larger than cross-effort ones, that is $\partial p_{1}(.) / \partial e_{1} \geq\left|\partial p_{2}(.) / \partial e_{1}\right|$. Furthermore, $\rho$ is an index for product differentiation. It also satisfies $|\rho| \leq 1$ to ensure that own-price effects are larger than cross-price ones in the direct demand system, that is $\left|\partial q_{i}(.) / \partial p_{i}\right| \geq\left|\partial q_{i}(.) / \partial p_{-i}\right|$. For expositional purposes, we focus on only two cases of interest. In the first one, market and effort externalities have the same sign, that is, in the parameter region where $\sigma \rho>0$. The second relevant region of parameters is that where $\sigma \rho \leq 0$, with $\sigma \geq 0$ and $\rho \leq 0$, which describes a free-riding context. ${ }^{8}$

Exerting effort is costly and, for simplicity, we assume that the corresponding disutility function for $R_{1}$ is quadratic, namely $\Psi\left(e_{1}\right)=\psi e_{1}^{2} / 2 .{ }^{9} \quad$ Finally, we assume that both upstream and downstream firms produce at constant marginal costs normalized to zero.

Incentive mechanisms: $S_{1}$ can use two different classes of incentive mechanisms: RPM or QF contracts. In each case, he offers menus of contracts to screen his retailer according to the realization of demand.

Under QF, for instance, an incentive mechanism is a menu of contracts of the form $\left\{t_{1}(\hat{\theta}), q_{1}(\hat{\theta})\right\}_{\hat{\theta} \in \Theta}$ where $\hat{\theta}$ is $R_{1}$ 's report on the demand parameter, $q_{1}(\hat{\theta})$ is the corresponding input level supplied by $S_{1}$ and $t_{1}(\hat{\theta})$ is the fixed-fee paid by $R_{1}$ to $S_{1}$.

Similarly, if RPM is chosen, an incentive mechanism is of form $\left\{t_{1}(\hat{\theta}), q_{1}(\hat{\theta}), p_{1}(\hat{\theta})\right\}_{\hat{\theta} \in \Theta}$ where now $p_{1}(\hat{\theta})$ denotes the retail price of good 1 following report $\hat{\theta}{ }^{10}$

\footnotetext{
${ }^{7}$ As it will become clear later on, since $S_{2}-R_{2}$ is vertically integrated, there is no loss of insights in assuming that $R_{2}$ does not exert any effort.

${ }^{8}$ Assuming that goods are complements, $\rho \geq 0$, and that effort creates negative externalities, $\sigma \leq 0$, seems unreasonable.

${ }^{9}$ In an earlier version, we made no assumption on disutility and on the distribution of types and get all results below when assuming that uncertainty is small by means of Taylor expansions.

${ }^{10}$ In our environment this is without loss of generality. Indeed, when $S_{1}$ no longer controls the level of final output sold in the market, but can only fix the retail price under RPM, the analysis remains the same as if output was observable. The argument is formally developed in Martimort and Piccolo (2007). The idea is that, for screening purposes, the optimal RPM mechanism reduces the input supply below what would be optimal under complete information. Indeed, consider the output choice of the non-integrated retailer when final output is non-verifiable. Since his objective is similar to that of an integrated structure under complete information, the retailer would like to expand output up to the point where the marginal benefit of one extra unit (the retail price) equals the marginal disutility of effort. Thus $R_{1}$ would like to
} 
A QF arrangement is less complete relative to RPM because it restricts the set of screening instruments available to the manufacturer by leaving unspecified the retail price. Therefore, QF arrangements amount to a vertically decentralized organizational structure. With such contractual scheme, the upstream manufacturer does not have enough instruments to monitor the promotional effort exerted by the retailer. Instead, by dictating the retail price and the quantity sold to the retailer, the upstream manufacturer is able to control directly the retailer's effort level under RPM.

Note that even a RPM arrangement is an incomplete contract. Indeed, manufacturer $S_{1}$ cannot contract on the output and retail price chosen by $S_{2}-R_{2}$. This assumption de facto rules out any "grand-contract" based on all information available downstream. It is justified either because such contracts are generally viewed as collusive practices by antitrust authorities, or because communication between a manufacturer and a retailer selling a competing product is not even feasible.

Contracts are secret and, following Roger Myerson (1982) and David Martimort (1996), we use a version of the Revelation Principle in competing hierarchies to characterize the set of incentive feasible allocations. Indeed, with secret contracts, for any output chosen by the integrated structure $S_{2}-R_{2}$, there is no loss of generality in looking for $S_{1}$ 's best response within the class of direct and truthful mechanisms to characterize pure strategy equilibria.

Commitment to vertical restraints: Since the mechanism ruling the hierarchy $S_{1}-R_{1}$ is private, it cannot have any commitment value vis-à-vis the vertically integrated structure $S_{2}-R_{2}$. This seems a quite natural assumption in environments where details of the vertical deals between a manufacturer and his retailer are rarely available to competitors.

However, the choice of the specific contractual mode, namely whether quantity forcing (QF) or resale price maintenance (RPM) is chosen by a given manufacturer, is itself observable by competing hierarchies. This commitment assumption plays a central role in our analysis and deserves some motivation.

The key issue is whether principals can credibly commit not to exert vertical price control. In practice, in OECD countries where exemptions to RPM practices are admitted, manufacturers are requested to ask Antitrust authorities to use these contracts and this move is publicly observable. ${ }^{11}$

More generally, there are other ways by which suppliers can commit themselves to choose a particular kind of vertical arrangements.

First, the observability of retail prices might require setting up some (observable) monitoring technology in advance, especially in cases where retailers may include either nonverifiable elements or secret price cuts with their customers. Not acquiring such technology at the outset makes gathering information on retail prices impossible afterwards. ${ }^{12}$

expand output above the second-best level implemented by our mechanism $\left\{t_{1}(),. q_{1}(),. p_{1}().\right\}$ irrespective of the sign of $\sigma \rho$. This implies that there is no incentives to sell a quantity lower than that supplied by the manufacturer and shows that our mechanism is robust to the lack of verifiability of the final output sold by the retailers. Including this quantity as an explicit contracting variables facilitates presentation.

${ }^{11} \mathrm{RPM}$ is generally prohibited in almost all OECD countries. Some countries though do have a procedure for authorizing this practice if the beneficial effects can be shown to outweigh the detrimental ones. See, for example, Alan E. Bollard (1989) as for New Zealand. Firms wishing to justify RPM, it is argued, should be asked to demonstrate the efficiency gains they claim rather than using scarce competition authority resources to establish the inefficiency of each system.

${ }^{12}$ In this respect, consider a simple extension of our model with several downstream retailers dealing 
Second, even if the retail price is easily observable, suppliers may build reputation for not using it as a contractual variable. Thus, commitment to QF leaves more freedom to downstream retailers and this may have also a positive feed-back in fostering downstream investments and initiative. In this respect, our model can also be viewed as a shortcut for a slightly more complex setting where not only retail prices and quantities may be contractually specified but also other input requirements. Freeing the agent from any control of those activities may have the same commitment value as giving up price control in our simplified model.

Third, a simple legal instrument, commonly used in business practices, which makes this commitment credible is to stipulate very high penalties for breaching any announced contractual mode. ${ }^{13}$

Timing, strategies and equilibrium concept: Firms play a three-stage game whose sequence of events unfolds as follows:

1. $S_{1}$ either publicly commits to verify the (ex post) realization of the retail price in market-1 (RPM), together with $R_{1}$ 's sales level or, alternatively, she might give up vertical control and use only sales as a screening device (QF).

2. Uncertainty about demand realizes and only $R_{1}$ and $S_{2}-R_{2}$ observe it.

3. $S_{1}$ secretly offers a menu of contracts of the chosen class to $R_{1}$. Once this menu has been accepted, $R_{1}$ picks one element within that menu by sending a message $\hat{\theta}_{1} \in \Theta$ to $S_{1}$ about the realized demand state. Effort is exerted, product market competition takes place and, finally, payments are made after verifiable actions have been observed. If the offer is turned down, $S_{1}$ and $R_{1}$ enjoy their outside options which are normalized to zero, and the integrated structure $S_{2}-R_{2}$ acts as a monopolist in the downstream market.

The equilibrium concept we use is Perfect Bayesian Equilibrium with the added "passive beliefs" refinement. Provided $R_{1}$ receives any unexpected offer from $S_{1}$, he still believes that $S_{2}-R_{2}$ produces the same equilibrium quantity. We denote by $\mathcal{G}$ the three stage game of contractual choices cum mechanisms offers and market interactions. We shall look for equilibria in pure strategies of $\mathcal{G}$.

Our analysis will be developed under the following assumption on parameter values: ${ }^{14}$

(A1)

$$
\psi>\max \left\{\frac{1}{2}, \frac{2+\sigma \rho}{4-2 \rho(\sigma+\rho)}, \frac{1+\sigma \rho}{2-\rho^{2}}\right\}
$$

As we will show below, there always exists a unique linear equilibrium of $\mathcal{G}$ as long as the distribution on demand shocks is uniform, the effort disutility is quadratic and inverse

with the same manufacturer and suppose that those retailers observe each other retail prices. A commitment to have exclusive territories may act as a device not to learn retail prices whereas intrabrand downstream competition between retailers may help revealing this information. Such commitment to choose either exclusive territories or instead intrabrand competition is certainly observable and credible. Patrick Rey and Joseph Stiglitz (1995) analyzed the commitment role of exclusive territories although in a model with public contracts.

${ }^{13}$ Of course, this argument is valid as long as nothing unexpected ever occurs.

${ }^{14}$ In the Appendix, we show that this assumption allows us to focus on fully separating allocations whatever the contractual arrangement under scrutiny. 
demands are linear as supposed. In such linear equilibria, efforts and quantities depend linearly on the demand shock $\theta \cdot{ }^{15}$

Complete information benchmark: For each realization of $\theta$ and any effort-output pair $\left\{e_{1}(\theta), q_{1}(\theta)\right\}_{\theta \in \Theta}$ implemented by $S_{1}$, the vertically integrated structure $S_{2}-R_{2}$ solves:

$$
q_{2}(\theta)=\underset{q_{2} \in \mathbb{R}_{+}}{\arg \max } p_{2}\left(\theta, e_{1}(\theta), q_{2}, q_{1}(\theta)\right) q_{2}=\frac{1}{2}\left(\theta+\rho q_{1}(\theta)+\sigma e_{1}(\theta)\right) .
$$

Under complete information, the vertical structure $S_{1}-R_{1}$ 's maximizes at each $\theta$ its vertically integrated profit:

$$
\left(e_{1}(\theta), q_{1}(\theta)\right)=\underset{\left(e_{1}, q_{1}\right) \in \mathbb{R}_{+}^{2}}{\arg \max }\left\{p_{1}\left(\theta, e_{1}, q_{1}, q_{2}(\theta)\right) q_{1}-\Psi\left(e_{1}\right)\right\} .
$$

which leads to the following first-order conditions:

$$
q_{1}(\theta)=\frac{1}{2}\left(\theta+\rho q_{2}(\theta)+e_{1}(\theta)\right) \text { and } q_{1}(\theta)=\psi e_{1}(\theta) .
$$

Solving equations (2) and (4) gives us the Nash equilibrium outputs and effort under complete information. For each $\theta$ let us denote by $\left\{p_{1}^{*}(\theta), q_{1}^{*}(\theta), e_{1}^{*}(\theta)\right\}$ the solution to the following equations:

$$
q_{1}^{*}(\theta)=\frac{(2+\rho) \psi \theta}{\psi\left(4-\rho^{2}\right)-2-\rho \sigma}, \quad q_{1}^{*}(\theta)=\psi e_{1}^{*}(\theta), \quad p_{1}^{*}(\theta)=q_{1}^{*}(\theta) .
$$

These are respectively the quantity, downstream level of effort and retail price for each realization of demand that $S_{1}$ would recommend to $R_{1}$ to maximize the profit of their vertical structure in a Nash equilibrium with the integrated hierarchy $S_{2}-R_{2}$.

Under complete information, the marginal cost of effort must be equal to own market sales which means that the retailer's choice of effort is aligned with that of the vertical structure he forms with the upstream manufacturer. Because there is no vertical externality under complete information, the effort level will be the same whether $S_{1}$ allows $R_{1}$ to choose his downstream effort or impose it through a secret contract. There is no way of affecting downstream competition. QF and RPM both implement the same outcome. This is no longer the case under asymmetric information since it induces a vertical externality. The agent's choice of effort is no longer aligned with that of his principal and the latter can use this conflict strategically to influence downstream competition.

\section{Equilibrium characterization}

Downstream moral hazard has generally two different effects in games of competing hierarchies. First, it will create an agency problem in the vertical structure even under RPM. Second, effort in enhancing own demand may have an impact on competitor's demand. RPM and QF may affect differently the demand faced by competing retailers and thus have a strategic value. In this framework, neither RPM nor QF allows $S_{1}$ to fully extract

\footnotetext{
${ }^{15}$ On that existence, see also Martimort (1996) who studied a related model (although without effort).
} 
$R_{1}$ 's information rents since, even when the retail price can be contracted upon, $S_{1}$ cannot disentangle the impact of the intercept parameter $\theta$ and the retailer's effort on the residual demand the latter faces. The possibility for the retailer to claim that large sales are due to a high effort level and a lower demand than what he has really observed, whereas they result instead from a higher demand and less effort, forces the upstream manufacturer to give up some information rent to high-demand retailers in order to induce truthtelling. As a result, the second-best allocation will be characterized by a downward distortion of both quantity and effort supplied by the retailer when he faces low demand states. This information rent, of course, depends on the chosen contractual mode.

In particular, the choice of the contractual mode has two opposite effects on $S_{1}$ 's profits whenever $R_{1}$ 's effort and output have the same impact on $S_{2}-R_{2}$ 's demand, namely $\rho \sigma>0$. On the one hand, restricting the set of screening instruments may lead the upstream supplier to grant more information rents relative to RPM: an agency cost effect. On the other hand, by changing the rivals' behavior at the market stage, QF may also have a strategic value relative to RPM: a strategic effect. The relative strength of these two effects will depend upon the severity of the agency problem.

As we shall prove, when demand uncertainty is small enough, the strategic effect dominates the agency one. Besides creating a vertical externality between $S_{1}$ and $R_{1}$ because, under asymmetric information, $S_{1}$ wants less downstream effort than what $R_{1}$ would like to exert, QF also generates an horizontal externality which may drive the integrated structure $S_{2}-R_{2}$ to behave in a more friendly manner. By contrast, in the freeriding set-up, the oversupply of effort provided by $R_{1}$ under QF makes his competitor more aggressive at the market stage so as to make the strategic effect reinforce the agency effect. QF is thus always dominated by RPM.

Below we solve the game in two steps. First, we characterize the market allocation under both contractual regimes. Then, the equilibrium contract will be derived by using a backward induction argument.

Note that $S_{2}-R_{2}$ 's best-response is still given by equation (2), i.e.,

$$
q_{2}(\theta)=\underset{q_{2} \in \mathbb{R}_{+}}{\arg \max } p_{2}\left(\theta, e_{1}(\theta), q_{2}, q_{1}(\theta)\right) q_{2}=\frac{1}{2}\left(\theta+\rho q_{1}(\theta)+\sigma e_{1}(\theta)\right) .
$$

One can already infer that $S_{1}$ has an incentive to choose strategically the contractual mode to influence $S_{2}-R_{2}$ 's behavior. By choosing QF, $S_{1}$ commits to let $R_{1}$ choose freely the demand-enhancing effort $e_{1}$. Because the retailer better internalizes the impact of his effort on his own demand, QF shifts $e_{1}$ up. ${ }^{16}$ When goods are complements $(\rho>0)$, shifting the $S_{2}-R_{2}$ 's reaction function up has a positive strategic effect when efforts have a cooperative value $(\sigma>0)$. Instead, when effort is selfish $(\sigma<0)$ and goods are substitutes $(\rho<0)$, shifting that reaction function down is certainly the best option.

RPM: In this regime $S_{1}$ can contract also on the retail price beside the quantity supplied by the downstream firm to final consumers. The effort level is then indirectly fixed as a function of $\theta$ through the inverse demand, i.e., $e_{1}=p_{1}+q_{1}-\rho q_{2}-\theta$. Intuitively, RPM is less flexible than QF simply because, when the retailer faces a retail price target, he is

\footnotetext{
${ }^{16}$ Everything else being kept constant.
} 
forced to choose a suboptimal effort level from his viewpoint. ${ }^{17}$

Let us define $R_{1}$ 's information rent as:

$$
U_{1}(\theta)=p_{1}(\theta) q_{1}(\theta)-\Psi\left(p_{1}(\theta)+q_{1}(\theta)-\rho q_{2}(\theta)-\theta\right)-t_{1}(\theta) .
$$

Incentive compatibility implies:

$$
U_{1}(\theta)=\max _{\hat{\theta} \in \Theta}\left\{p_{1}(\hat{\theta}) q_{1}(\hat{\theta})-\Psi\left(p_{1}(\hat{\theta})+q_{1}(\hat{\theta})-\rho q_{2}(\theta)-\theta\right)-t_{1}(\hat{\theta})\right\} .
$$

This yields the following first- and second-order local conditions for incentive compatibility: $:^{18}$

$$
\dot{U}_{1}(\theta)=\left(1+\rho \dot{q}_{2}(\theta)\right) \psi e_{1}(\theta),
$$

and

$$
\left(\dot{p}_{1}(\theta)+\dot{q}_{1}(\theta)\right)\left(1+\rho \dot{q}_{2}(\theta)\right) \geq 0,
$$

which, together with the participation constraint

$$
U_{1}(\theta) \geq 0
$$

define the set of incentive feasible allocations in $S_{1}-R_{1}$ hierarchy for a fixed output schedule $q_{2}(\theta)$ chosen by the rival pair $S_{2}-R_{2}$.

$S_{1}$ 's problem $\left(\mathcal{P}^{P}\right)$ is to design a menu of contracts to maximize the expected franchise fee he receives from $R_{1}$ subject to participation and incentive compatibility constraints, together with the additional restriction required by the retail price target. Expressing the fixed fee $t_{1}(\theta)$ as a function of the retailer's revenue and information rent, $\left(\mathcal{P}^{P}\right)$ becomes

$$
\left(\mathcal{P}^{P}\right): \max _{\left\{U_{1}(\cdot), q_{1}(\cdot), e_{1}(\cdot)\right\}} \int_{\underline{\theta}}^{\bar{\theta}}\left\{\left(\theta+e_{1}(\theta)+\rho q_{2}(\theta)-q_{1}(\theta)\right) q_{1}(\theta)-\psi \frac{e_{1}^{2}(\theta)}{2}-U_{1}(\theta)\right\} d \theta
$$

$$
\text { subject to (5), (6) and (7). }
$$

We will first assume and check ex post that $1+\rho \dot{q}_{2}(\theta) \geq 0$ for all $\theta$. Then $U_{1}(\theta)$ is increasing and the retailer's participation constraint (7) binds only for the lowest level of demand $\underline{\theta}$. We obtain immediately:

$$
U_{1}(\theta)=\int_{\underline{\theta}}^{\theta}\left(1+\rho \dot{q}_{2}(x)\right) \psi e_{1}(x) d x .
$$

Using this expression to compute the expected rent left to $R_{1}$, inserting into the maximand of $S_{1}$ 's problem, integrating by parts and neglecting (6) (that will be checked ex post also) yields a relaxed optimization problem $\left(\mathcal{P}^{P \prime}\right)$ :

$\max _{\left\{q_{1}(\cdot), e_{1}(\cdot)\right\}} \int_{\underline{\theta}}^{\bar{\theta}}\left\{\left(\theta+e_{1}(\theta)-q_{1}(\theta)+\rho q_{2}(\theta)\right) q_{1}(\theta)-\psi \frac{e_{1}^{2}(\theta)}{2}-(\bar{\theta}-\theta)\left(1+\rho \dot{q}_{2}(\theta)\right) \psi e_{1}(\theta)\right\} d \theta$.

\footnotetext{
${ }^{17}$ Under retail price restrictions the upstream producer has full control of all available instruments. See also Blair and Lewis (1994) and Martimort and Piccolo (2007) for related analysis.

${ }^{18}$ In the Appendix, we show that those local conditions are also sufficient for global optimality of the truthtelling strategy.
} 
At a best-response to the schedule $q_{2}(\theta)$ implemented by the competing pair $S_{2}-R_{2}$, the production and effort in $S_{1}-R_{1}$ hierarchy are respectively given by the following first-order conditions obtained by pointwise optimization: ${ }^{19}$

$$
\begin{gathered}
q_{1}(\theta)=p_{1}(\theta)=\theta+e_{1}(\theta)+\rho q_{2}(\theta)-q_{1}(\theta), \\
q_{1}(\theta)=\psi\left(e_{1}(\theta)+(\bar{\theta}-\theta)\left(1+\rho \dot{q}_{2}(\theta)\right)\right) .
\end{gathered}
$$

Under RPM, the only variable which is really useful to reduce $R_{1}$ 's information rent is his own effort as it can be seen on (5). Hence, this effort level needs to be downward distorted relative to its complete information level. At the same time, the pricing rule is unchanged and keeps the same expression as under complete information as seen from (8). Output is produced according to the efficient rule conditionally on a given effort but this effort is distorted downward under asymmetric information. ${ }^{20}$

By using (2) together with (8) and (9), one can check that the allocation $\left\{e_{1}^{P}(\theta), q_{1}^{P}(\theta)\right\}_{\theta \in \Theta}$ solves the following system of differential equations:

$$
\begin{gathered}
\dot{q}_{1}^{P}(\theta)=\frac{2\left(q_{1}^{P}(\theta)-\psi e_{1}^{P}(\theta)\right)-(\bar{\theta}-\theta) \psi\left(2+\rho\left(1+\sigma \dot{e}_{1}^{P}(\theta)\right)\right)}{\rho^{2}(\bar{\theta}-\theta) \psi}, \\
e_{1}^{P}(\theta)=\frac{\left(4-\rho^{2}\right) q_{1}^{P}(\theta)-(2+\rho) \theta}{2+\rho \sigma}
\end{gathered}
$$

with the boundary conditions $q_{1}^{P}(\bar{\theta})=q_{1}^{*}(\bar{\theta})$ and $e_{1}^{P}(\bar{\theta})=e_{1}^{*}(\bar{\theta})$.

In the Appendix, we show that, under A1, the equilibrium output is always downwards distorted. More precisely, the ability of a retailer to pretend having observed a slightly lower level of demand forces the supplier to give up a rent in order to elicit information revelation. To reduce this costly rent, the supplier reduces effort below its complete information level. Although sales are not used for rent extraction purposes, output itself has to fall below its complete information level because effort is downward distorted, but this effect is indirect only.

QF: Now $S_{1}$ no longer contracts on the retail price, but she still observes and contracts on the quantity supplied by $R_{1}$ on the retail market.

Let us now redefine retailer $R_{1}$ 's information rent under a QF regime as:

$$
U_{1}(\theta)=-t_{1}(\theta)+\max _{e_{1} \in \mathbb{R}_{+}}\left(\theta+e_{1}-q_{1}(\theta)+\rho q_{2}(\theta)\right) q_{1}(\theta)-\Psi\left(e_{1}\right) .
$$

Incentive compatibility implies now: ${ }^{21}$

$$
U_{1}(\theta)=\max _{\hat{\theta} \in \Theta}\left\{-t_{1}(\hat{\theta})+\max _{e_{1} \in \mathbb{R}_{+}}\left(\theta+e_{1}-q_{1}(\hat{\theta})+\rho q_{2}(\theta)\right) q_{1}(\hat{\theta})-\Psi\left(e_{1}\right)\right\} .
$$

From which we obtain the following first- and second-order local conditions for incentive compatibility:

$$
\dot{U}_{1}(\theta)=\left(1+\rho \dot{q}_{2}(\theta)\right) q_{1}(\theta),
$$

\footnotetext{
${ }^{19}$ Given concavity of the objective, these conditions are also sufficient.

${ }^{20}$ This is reminiscent of the so-called 'dichotomy' between pricing rule and incentives found in a regulatory environment with a single hierarchy (Jean-Jacques Laffont and Jean Tirole, 1993, Ch. 3).

${ }^{21}$ Again, those local conditions are proved to be sufficient also in the Appendix.
} 


$$
\left(1+\rho \dot{q}_{2}(\theta)\right) \dot{q}_{1}(\theta) \geq 0 .
$$

Finally, because the agent is residual claimant for any impact of his demand-enhancing effort under a QF arrangement, we have:

$$
q_{1}(\theta)=\psi e_{1}(\theta)
$$

Taking into account this latter expression of $R_{1}$ 's effort, $S_{1}$ 's contracting problem can be written as:

$$
\left(\mathcal{P}^{Q}\right): \max _{\left\{U_{1}(\cdot), q_{1}(\cdot)\right\}} \int_{\underline{\theta}}^{\bar{\theta}}\left\{\left(\theta+\frac{q_{1}(\theta)}{\psi}+\rho q_{2}(\theta)-q_{1}(\theta)\right) q_{1}(\theta)-\frac{q_{1}^{2}(\theta)}{2 \psi}-U_{1}(\theta)\right\} d \theta
$$

$$
\text { subject to (12), (13) and (7). }
$$

For any given quantity schedule specified by the direct revelation mechanism QF, $R_{1}$ gains flexibility under a quantity-fixing arrangement since he chooses now optimally his effort level. More specifically, while choosing the optimal effort level, the retailer does not internalize the impact of his effort on the information rent given up by the upstream manufacturer. QF introduces a vertical externality between the manufacturer and his retailer. As rents and effort are positively related via quantity, it will be thus profitable to oversupply effort relative to RPM everything else being kept equal.

We again first assume and check ex post that $1+\rho \dot{q}_{2}(\theta) \geq 0$ for all $\theta$. Then $U_{1}(\theta)$ is increasing and the participation constraint binds at $\underline{\theta}$ only. We obtain immediately:

$$
U_{1}(\theta)=\int_{\underline{\theta}}^{\theta}\left(1+\rho \dot{q}_{2}(x)\right) q_{1}(x) d x
$$

Using this expression to compute the expected rent left to $R_{1}$, integrating by parts and inserting into the maximand of $\left(\mathcal{P}^{Q}\right)$ yields the expression of the relaxed program $\left(\mathcal{P}^{Q^{\prime}}\right)$ :

$$
\max _{\left\{q_{1}(\cdot)\right\}} \int_{\underline{\theta}}^{\bar{\theta}}\left\{\left(\theta+\frac{q_{1}(\theta)}{\psi}+\rho q_{2}(\theta)-q_{1}(\theta)\right) q_{1}(\theta)-\frac{q_{1}^{2}(\theta)}{2 \psi}-(\bar{\theta}-\theta)\left(1+\rho \dot{q}_{2}(\theta)\right) q_{1}(\theta)\right\} d \theta .
$$

At a best-response to the schedule $q_{2}(\theta)$ implemented by the pair $S_{2}-R_{2}$, we get:

$$
\theta+\frac{\left.q_{1}^{Q}(\theta)\right)}{\psi}-2 q_{1}^{Q}(\theta)+\rho q_{2}^{Q}(\theta)-(\bar{\theta}-\theta)\left(1+\rho \dot{q}_{2}^{Q}(\theta)\right)=0 .
$$

Differentiating equation (2) yields $2 \dot{q}_{2}^{Q}(\theta)=1+\rho \dot{q}_{1}^{Q}(\theta)+\sigma \dot{e}_{1}^{Q}(\theta)$, using this condition together with $\psi \dot{e}_{1}^{Q}(\theta)=\dot{q}_{1}^{Q}(\theta)$, one can immediately show that $q_{1}^{Q}(\theta)$ solves the following differential equation:

$$
\dot{q}_{1}^{Q}(\theta)=\frac{\psi(2+\rho)(2 \theta-\bar{\theta})+\left(2+\rho \sigma-\psi\left(4-\rho^{2}\right)\right) q_{1}^{Q}(\theta)}{\rho(\bar{\theta}-\theta)(\sigma+\psi \rho)},
$$

with the boundary condition $q_{1}^{Q}(\bar{\theta})=q_{1}^{*}(\bar{\theta})$.

Under $\mathrm{QF}, S_{1}$ gives up a screening instrument by not controlling the retail price. The only remaining screening device is output which must be downward distorted for 
rent extraction reasons. Contrary to the case of RPM, the pricing rule is now distorted compared with the complete information and the RPM cases.

Once again, the ability of a retailer having observed a given shock on demand to pretend demand was slightly lower forces the supplier to give up a costly information rent. Reducing this rent requires a downward distortion of the output level below its complete information level, i.e., $q_{1}^{Q}(\theta)<q_{1}^{*}(\theta)$ for all $\theta<\bar{\theta}$. Moreover, although effort is now chosen efficiently by the retailer, the fact that sales are downward distorted implies that the effort itself falls below its first-best level, but this effect is again indirect only.

\section{The strategic value of incomplete contracts}

Having characterized the market allocation under both contractual regimes, we now turn to investigate whether RPM or QF is the preferred contractual mode at equilibrium. As a preliminary result, the next Proposition provides a useful description of how outputs and efforts are ordered under both regimes. This result will be key for showing, as well as interpreting, the equilibrium characterization provided below.

Proposition 1 Assume that $\boldsymbol{A} 1$ holds. The following properties are satisfied:

- $e_{1}^{Q}(\theta) \geq e_{1}^{P}(\theta)$ for all $\theta$ with equality holding only at $\bar{\theta}$;

- $q_{2}^{Q}(\theta) \geq q_{2}^{P}(\theta)$ for all $\theta$ with equality only at $\bar{\theta}$ (resp. $\leq$ ) if and only if $\sigma>0$ (resp. $<)$;

- $q_{1}^{Q}(\theta) \geq q_{1}^{P}(\theta)$ for all $\theta$ with equality only at $\bar{\theta}($ resp. $\leq)$ if and only if $\rho \sigma>0$ (resp. $<)$.

When the upstream manufacturer gives up control on retail price, the downstream firm increases his information rent by playing on his effort choice. Under QF, $R_{1}$ chooses his effort according to the efficient rule (14). Thus, $R_{1}$ finds it profitable to supply more effort under a QF contract relative to RPM in order to enjoy more rent. Hence $e_{1}^{Q}(\theta)$ must be larger than $e_{1}^{P}(\theta)$. This difference in efforts shifts in turn the reaction function of the competing integrated structure when moving from RPM to QF. From equation (2) it is easy to check that, when $\sigma>0$, the output level $q_{2}(\theta)$ increases with such a move, and it diminishes otherwise. This explains why besides introducing a vertical externality between $S_{1}$ and $R_{1}$, QF also creates an horizontal externality on the integrated structure $S_{2}-R_{2}$, so that $q_{2}^{Q}(\theta)$ is larger than $q_{2}^{P}(\theta)$ when $\sigma>0$, and lower when $\sigma<0$.

Finally, as for $q_{1}(\theta)$, three different effects are at play simultaneously by moving from RPM to QF. First, for any given output level, $R_{1}$ will exert more effort under QF relative to RPM. When the retail price is not controlled, the agent is residual claimant for the full impact of his effort on enhancing demand. This effect raises effort and thus $R_{1}$ 's output: a demand-enhancing effect. Second, since sales are the only screening instrument under QF, $S_{1}$ needs to distort it downward for rent extraction reasons: a rent extraction effect. Third, owing to the horizontal externality, the output of the competing structure $S_{2}-R_{2}$ 
is shifted upward when goods are substitutes and downward when they are complements: a strategic effect. ${ }^{22}$

Yet, when products are differentiated and efforts generate demand spillovers, the strategic effect gets stronger when market and effort externalities have the same sign, that is $\rho \sigma>0$. In this case, $\mathrm{QF}$ increases effort and moves $q_{2}(\theta)$ in the right direction. By contrast, in the free-riding case the strategic effect leads the integrated structure $S_{2^{-}}$ $R_{2}$ to behave more aggressively at the market stage since the consumers' willingness to pay increases and $q_{2}(\theta)$ increases. This in turn lowers $q_{1}(\cdot)$ when quantities are strategic substitutes.

Armed with this characterization, we can now show the main result of the paper. In the next theorem we provide conditions, related to the presence of externalities between agents, under which QF has a strategic value relative to RPM.

Theorem 1 Assume that $\boldsymbol{A} 1$ holds. Then, as long as $\Delta \theta$ is small enough, $S_{1}$ prefers $Q F$ if $\rho \sigma>0$, and $R P M$ when $\rho \sigma \leq 0$.

The economic intuition of this result is simple and builds upon the insights provided by Proposition 1. By inducing more effort on the retailer's side, QF changes also the market behavior of the competing structure. Of course, once one moves from RPM to $\mathrm{QF}$, information rents increase because a screening instrument is given up: an agency cost effect. When goods are independent or effort does not create any horizontal externalities between retailers, this agency cost leads the upstream supplier to always prefer RPM. If, instead, goods are differentiated and there are effort spillovers, the strategic effect may outweigh the extra agency costs associated to QF whenever market and non-market externalities have the same sign and the cost of asymmetric information is not very large, that is $\Delta \theta$ is small.

In fact, as QF allows $S_{1}$ to force $S_{2}-R_{2}$ to behave in a more friendly manner at the market stage, it raises the supplier's profits by increasing effort and so the expected transfer that can be extracted from $R_{1}$. In the free-riding case, though, the strategic effect has a negative value as QF makes $S_{2}-R_{2}$ more aggressive relative to RPM. This adds to the excessive agency costs effect and thus leads $S_{1}$ to prefer RPM.

Remark 1: It is important to notice that the insight delivered by Theorem 1 extends directly to a more general framework with two (symmetric) competing hierarchies (see Kastl, Martimort and Piccolo, 2008). Both the agency cost effect and the strategic effect illustrated above survive in such a model, and are key to show that choosing QF is an equilibrium of the game in the limit of small uncertainty whenever $\sigma \rho>0$. Indeed, a QF contract still leaves more possibilities for the agent to grasp information rent, but for any given class of mechanisms ruling the competing hierarchy, restricting the set of the screening instruments available to a principal may create a strategic effect influencing the rival's market behavior. As before, the vertical externality that QF creates within each vertical hierarchy is translated horizontally on the competing organization. Increasing

\footnotetext{
${ }^{22}$ When efforts do not create externalities, $\sigma=0$, or goods are independent, $\rho=0$, the latter strategic effect is absent. The demand-enhancing and the rent extraction effects then exactly compensate in our quadratic framework, that is $q_{1}^{Q}(\theta)=q_{1}^{P}(\theta)$. As a consequence, the competing hierarchies framework at hand displays the same features as the sequential monopolies model studied in Martimort and Piccolo (2007).
} 
retailers' effort thus provides a beneficial effect on supplier's profits to the extent that it weakens the competitive stance of the opposing hierarchy on the downstream market.

Remark 2: It is worth closing this Section with a general comment on our methodology. It could be argued that the simple result that less complete contracts could have a strategic value can be obtained in a simpler complete information model. Consider the case where demand is common knowledge and $S_{1}$ either uses a two-part tariff or a linear wholesale price. Two-part tariffs imply fierce downstream competition. Linear prices commit instead the retailer to decrease his output downstream, which may have a positive effect in the case of demand complements or price competition. ${ }^{23}$ However, linear pricing performs badly in shifting profits upstream so that part of the strategic gains of using them cannot be recouped upstream. The methodological issue is that, under complete information, there is no reason a priori to restrict the use of fixed-fees as a mean of extracting the retailer's downstream profit. Asymmetric information, instead, endogenizes the limits on the ability of upstream manufacturer to capture downstream profit and provides better foundations for the possible restrictions on instruments associated to different vertical restraints.

\section{$5 \quad$ Related literature}

Our paper belongs to three strands of literature with some overlap: the literature on the strategic design of incomplete contracts, the literature on vertical restraints, and lastly the literature on strategic delegation.

Strategic design of incomplete contracts: The idea that, in a strategic situation, reducing a player's set of actions or allowing him to commit to suboptimal actions may increase his payoff has already been studied. In the incomplete contracts literature, this insight leads to show that "more incompleteness" may help to improve contractual outcomes. To date, the literature has shown that it can be done either by relaxing dynamic incentive constraints or because it facilitates signalling of private information.

Concerning the role of incompleteness in dynamic environments, Mathias Dewatripont and Eric Maskin (1990, 1995) and Jacques Crémer (1995) showed that a principal might voluntarily limit the set of variables used to contract with his agent to relax renegotiation constraints. ${ }^{24}$ Klaus M. Schmidt and Monika Schnitzer (1995), Douglas B. Bernheim and Michael D. Whinston (1998) and David Pearce and Ennio Stacchetti (1998) analyzed complete information models where nonverifiable actions can only be enforced through repeated interactions. Writing less complete contracts may relax incentive constraints in those repeated games.

Philippe Aghion and Benjamin Hermalin (1990), Franklin Allen and Douglas Gale (1992) and Kathryn Spier (1992) stressed the signalling value of incomplete contracts. When contracts can only be signed once principals are informed, incompleteness may

\footnotetext{
${ }^{23}$ A related effect is actually at work in Rey and Stiglitz (1995) although the commitment to increase retail prices comes there from the choice of exclusive territories for the retailers of the same manufacturer.

${ }^{24}$ Olsen and Torsvik (1993) and Martimort (1999) demonstrated also how moving away from a centralized regulation by introducing competing regulators, another form of incompleteness, may improve intertemporal commitment.
} 
be profitably used by a privately informed principal to credibly convey information to agents. $^{25}$

The strategic value of incomplete contracts stressed in our paper is novel and different from those above. The bilateral contract between a principal and his agent in a given hierarchy does not exert any externality on their selves as in a dynamic context but instead on competing retailers in a static game.

Vertical restraints: Our analysis is also related to the literature on vertical restraints under informational asymmetries (Piccolo et al., 2008, Gal-Or, 1991a, 1991b, 1991c, 1991d, 1999, Martimort, 1996, and Rey and Tirole, 1986, among others). While these previous contributions have mainly taken the set of control instruments available to a manufacturer as given, we endogenize this set in a strategic context. ${ }^{26}$ From an organizational design viewpoint, our results also explain why upstream manufacturers delegate non-market decisions, such as advertising and marketing activities, to downstream retailers (Lafontaine and Slade, 1997, and Andrea Sheppard, 1993).

Closer in spirit to our work, Bernard Caillaud and Patrick Rey (1995) started investigating information structures in producers-retailers hierarchies. Ignorance on the retailer's cost function might create a strategic advantage that could outweigh the associated agency costs. They focused on the value of ignorance in environments where principals can choose whether to acquire (at no additional costs) all the relevant market information or, alternatively, to stay (strategically) uninformed. In our set-up full extraction is prevented by a moral hazard component on non-market activities. This assumption is key for equilibria to display the less complete QF arrangements.

Strategic delegation: Our analysis also pertains to the literature dealing with strategic delegation and decentralized decision-making in competing hierarchies environments. However, it departs from it in two important respects: asymmetric information and secret contracting. First, the complete information literature on delegation has mainly focused on the strategic value of delegating choices to agents in contexts where the vertical structure between a principal and his agent has no raison-d'être a priori (Chaim Fershtman and Kenneth L. Judd, 1987, Chaim Fershtman, Kenneth L. Judd and Ehud Kalai, 1991, Steven Sklivas, 1987, among many others). Instead, in our model the privileged access of agents to information provides a rationale for the vertical structure in the first place. To analyze the corresponding asymmetric information between manufacturers and retailers, we follow Gal-Or (1991a), Martimort (1996) and Kai-Uwe Kuhn (1997) and consider a broader contract space (namely menus of contracts) than the simple linear or piecewise contracts used so far in the complete information literature on strategic delegation. Menus are flexible to fit different realizations of demand. Second, we focus on secret contracts. The terms of trade specified in vertical contracts are secret and cannot be used for strategic purposes; only the decision to use QF or RPM is public, an admittedly weaker assumption. Under asymmetric information, choosing a QF contract induces more information rent downstream but it has also a strategic value because more delegation affects downstream competition. ${ }^{27}$

\footnotetext{
${ }^{25}$ A similar idea has been applied by Roberta Dessi (2007). She shows that, in a pure moral hazard framework, incomplete contracts can be used as a foreclosure device via their signaling effects on entrants.

${ }^{26}$ Exceptions are Piccolo et al. (2008) and Martimort and Piccolo (2007), which however do not investigate when more incomplete contracts emerge spontaneously in a static game of competing hierarchies.

${ }^{27}$ See also Michael Katz (1991) and Bernard Caillaud, Bruno Jullien and Pierre Picard (1995) on the
} 


\section{Concluding remarks}

Focusing on a simple manufacturer-retailer model, we have shown that the equilibrium determinants of the choice between QF and RPM rest upon two somewhat natural aspects of information asymmetries: (i) the way different screening instruments shape agency costs; (ii) the type of externalities that bilateral negotiations between manufacturer-retailer pairs impose on competing vertical structures. The main result is that manufacturers dealing with (exclusive) competing retailers, may prefer to leave contracts silent on some (potentially) verifiable performance measures, namely price, whenever certain other aspects of agents' activity remain noncontractible. The key idea is that by allowing agents to respond more efficiently to competition, QF has a strategic value in that it may induce a more friendly behavior in the downstream competition. An interesting, and somewhat wider lesson of this result is that competition, and especially the channels through which it develops, can be an important source of contractual incompleteness.

\section{Appendix}

\subsection{Market equilibrium under RPM}

In this section we provide a formal characterization of the market equilibrium when $S_{1}$ offers RPM. The next lemma summarizes the results.

Lemma 1 Assume that $\boldsymbol{A} 1$ holds. Then the following properties are satisfied:

- $e_{1}^{P}(\theta) \leq e_{1}^{*}(\theta)$ and $q_{1}^{P}(\theta) \leq q_{1}^{*}(\theta)$ for all $\theta$ (with equality holding only at $\bar{\theta}$ )

- Information rents are increasing in $\theta$ and (6) holds.

Proof. We focus on linear equilibria where effort and quantities are linear in $\theta$, that is: $q_{1}^{P}(\theta)=q_{1}^{*}(\bar{\theta})-\dot{q}_{1}^{P}(\bar{\theta}-\theta)$ and $e_{1}^{P}(\theta)=e_{1}^{*}(\bar{\theta})-\dot{e}_{1}^{P}(\bar{\theta}-\theta)$ for all $\theta$ where $\dot{q}_{1}^{P}$ and $\dot{e}_{1}^{P}$ are two constants. From $(2), q_{2}^{P}(\theta)$ is also linear in $\theta, q_{2}^{P}(\theta)=q_{2}^{*}(\bar{\theta})-\dot{q}_{2}^{P}(\bar{\theta}-\theta)$ with $\dot{q}_{2}^{P}=\frac{1}{2}\left(1+\rho \dot{q}_{1}^{P}+\sigma \dot{e}_{1}^{P}\right)$.

To find the value of $\dot{q}_{1}^{P}$ and show that $\dot{q}_{1}^{P}>0$ when $\mathbf{A} \mathbf{1}$ holds, we differentiate w.r.t. $\theta$ equation (10) taking into the linearity of $q_{1}^{P}(\theta)$ and $e_{1}^{P}(\theta)$ :

$$
\dot{q}_{1}^{P}=\frac{-2\left(\dot{q}_{1}^{P}-\psi \dot{e}_{1}^{P}\right)+\psi\left(2+\rho\left(1+\sigma \dot{e}_{1}^{P}\right)\right)}{\rho^{2} \psi} .
$$

Using now (11) and differentiating w.r.t. $\theta$ to express $\dot{e}_{1}^{P}$ as a function of $\dot{q}_{1}^{P}$, and inserting into (17) yields:

$$
\dot{q}_{1}^{P}=\frac{2 \psi(2+\rho)}{\psi(4-2 \rho(\sigma+\rho))-\sigma \rho-2} .
$$

Therefore, $\dot{q}_{1}^{P}>0$ when

$$
\psi>\frac{2+\sigma \rho}{4-2 \rho(\sigma+\rho)}
$$

strategic value of secret contracts in vertical relationships plagued with agency problems. 
which is guaranteed by $\mathbf{A} \mathbf{1}$.

By using the definition of $q_{1}^{*}(\theta)$ we get:

$$
\dot{q}_{1}^{*}=\frac{\psi(2+\rho)}{\psi\left(4-\rho^{2}\right)-\sigma \rho-2}>0
$$

when A1 holds.

Now, since $q_{1}^{P}(\theta)-q_{1}^{*}(\theta)=\left(\dot{q}_{1}^{*}-\dot{q}_{1}^{P}\right)(\bar{\theta}-\theta)$ for all $\theta$, we get:

$$
\dot{q}_{1}^{P}-\dot{q}_{1}^{*}=\frac{(2+\sigma \rho)(2 \psi-1)(2+\rho) \psi}{(\psi(4-2 \rho(\sigma+\rho))-\sigma \rho-2)\left(\psi\left(4-\rho^{2}\right)-\sigma \rho-2\right)}
$$

which immediately implies $\dot{q}_{1}^{*}<\dot{q}_{1}^{P}$ by A1. Hence $q_{1}^{P}(\theta) \leq q_{1}^{*}(\theta)$ for all $\theta$ with equality only at $\bar{\theta}$.

By using the same kind of arguments, we have:

$$
\dot{e}_{1}^{P}=\frac{(2 \psi+1)(2+\rho)}{\psi(4-2 \rho(\sigma+\rho))-\sigma \rho-2} .
$$

Then, since $\psi \dot{e}_{1}^{*}=\dot{q}_{1}^{*}$, one gets:

$$
\dot{e}_{1}^{P}-\dot{e}_{1}^{*}=\frac{2(2+\rho)\left(4-\rho^{2}\right) \psi(2 \psi-1)}{(\psi(4-2 \rho(\sigma+\rho))-\sigma \rho-2)\left(\psi\left(4-\rho^{2}\right)-\sigma \rho-2\right)},
$$

implying $\dot{e}_{1}^{*}<\dot{e}_{1}^{P}$ by $\mathbf{A} \mathbf{1}$ and, since $e_{1}^{P}(\bar{\theta})=e_{1}^{*}(\bar{\theta}), e_{1}^{P}(\theta) \leq e_{1}^{*}(\theta)$ for all $\theta \leq \bar{\theta}$.

Using the expressions above of $\dot{q}_{1}^{P}$ and $\dot{e}_{1}^{P}$, we obtain:

$$
1+\rho \dot{q}_{2}^{P}=\frac{(2+\rho)(2 \psi-1)}{\psi(4-2 \rho(\sigma+\rho))-\sigma \rho-2}>0
$$

when A1 holds.

Notice also that program $\left(\mathcal{P}^{P \prime}\right)$ displays interior non-negative solutions when $\underline{\theta}$ is high enough. Together with (19), this implies that the information rent $U_{1}^{P}(\theta)$ is increasing as was supposed since

$$
0<\dot{U}_{1}^{P}(\theta)=\frac{(2+\rho)(2 \psi-1) \psi e_{1}^{P}(\theta)}{\psi(4-2 \rho(\sigma+\rho))-\sigma \rho-2} .
$$

Together with $\dot{q}_{1}^{P}>0,(19)$ also implies that the second-order conditions of the retailer $R_{1}$ 's problem, namely (6), hold since

$$
\left(\dot{q}_{1}^{P}(\theta)+\dot{p}_{1}^{P}(\theta)\right)\left(1+\rho \dot{q}_{2}^{P}(\theta)\right)=2 \dot{q}_{1}^{P}\left(1+\rho \dot{q}_{2}^{P}\right)>0 .
$$

Finally, let $U_{1}^{P}(\theta, \hat{\theta})$ be $R_{1}$ 's profits when the true retailer's type is $\theta$ but he announces a message $\hat{\theta} \neq \theta$ to $S_{1}$. To show that global incentive compatibility constraints hold we must have $\Gamma^{P}(\theta, \hat{\theta}) \equiv U_{1}^{P}(\theta, \theta)-U_{1}^{P}(\theta, \hat{\theta}) \geq 0$ for each pair $(\theta, \hat{\theta}) \in \Theta^{2}$. Simple algebraic manipulations taking into account that $p_{1}^{P}(\theta)=q_{1}^{P}(\theta)$ for all $\theta$, allow to rewrite $\Gamma^{P}(\cdot)$ as $\Gamma^{P}(\theta, \hat{\theta})=\int_{\hat{\theta}}^{\theta}\left\{2 \dot{q}_{1}^{P}(s) q_{1}^{P}(s)-\dot{t}_{1}^{P}(s)-2 \psi e_{1}^{P}(s, \theta) \dot{q}_{1}^{P}(s)\right\} d s$, where $e_{1}^{P}(s, \theta) \equiv 2 q_{1}^{P}(s)-$ $\rho q_{2}^{P}(\theta)-\theta$. By using (5) and substituting for $\dot{t}_{1}^{P}(s) \equiv 2\left(q_{1}^{P}(s)-\psi e_{1}^{P}(s)\right) \dot{q}_{1}^{P}(s)$ into $\Gamma^{P}(\cdot)$, one obtains:

$$
\Gamma^{P}(\theta, \hat{\theta})=2 \psi \int_{\hat{\theta}}^{\theta} \dot{q}_{1}^{P}(s)\left\{\int_{s}^{\theta}\left(1+\rho \dot{q}_{2}^{P}(x)\right) d x\right\} d s=\psi \dot{q}_{1}^{P}\left(1+\rho \dot{q}_{2}^{P}\right)(\theta-\hat{\theta})^{2} \geq 0 \quad \forall(\theta, \hat{\theta}) .
$$

where the last equality follows from the fact that the equilibrium is linear in types and the final inequality follows from (20) which concludes the proof. 


\subsection{Market equilibrium under QF}

We provide now a formal characterization of the market equilibrium when $S_{1}$ offers a $\mathrm{QF}$ contract. The next lemma summarizes the results.

Lemma 2 Assume that $\boldsymbol{A} 1$ holds. The following properties are satisfied:

- $e_{1}^{Q}(\theta) \leq e_{1}^{*}(\theta)$ and $q_{1}^{Q}(\theta) \leq q_{1}^{*}(\theta)$ for all $\theta$ (with equality only at $\bar{\theta}$ );

- Information rents are increasing in $\theta$ and (13) holds.

Proof. As before, we focus on a linear equilibrium where $q_{1}^{Q}(\theta)=q_{1}^{*}(\bar{\theta})-\dot{q}_{1}^{Q}(\bar{\theta}-\theta)$ for all $\theta$. We still have also $e_{1}^{Q}(\theta)$ and $q_{2}^{Q}(\theta)$ linear, with $\dot{q}_{2}^{Q}=\frac{1}{2}\left(1+\rho \dot{q}_{1}^{Q}+\sigma \dot{e}_{1}^{Q}\right)$, and $e_{1}^{Q}(\theta)=e_{1}^{*}(\bar{\theta})-\dot{e}_{1}^{Q}(\bar{\theta}-\theta)$. However, we have now, $\psi e_{1}^{Q}(\theta)=q_{1}^{Q}(\theta)$ for all $\theta$ and thus $\psi \dot{e}_{1}^{Q}=\dot{q}_{1}^{Q}$.

Taking into account this linearity and differentiating (16) with respect to $\theta$ yields:

$$
\dot{q}_{1}^{Q}=\frac{-2(2+\rho) \psi+\left(\left(4-\rho^{2}\right) \psi-(2+\rho \sigma)\right) \dot{q}_{1}^{Q}}{\rho(\sigma+\psi \rho)} .
$$

Simplifying, we get:

$$
\dot{q}_{1}^{Q}=\frac{(2+\rho) \psi}{\psi\left(2-\rho^{2}\right)-\sigma \rho-1}
$$

with $\dot{q}_{1}^{Q}>0$ when

$$
\psi>\frac{1+\sigma \rho}{2-\rho^{2}}
$$

which follows from A1.

We then have $q_{1}^{Q}(\theta)-q_{1}^{*}(\theta)=\left(\dot{q}_{1}^{*}-\dot{q}_{1}^{Q}\right)(\bar{\theta}-\theta)$ for all $\theta$ where:

$$
\dot{q}_{1}^{Q}-\dot{q}_{1}^{*}=\frac{(2+\rho)(2 \psi-1) \psi}{\left(\psi\left(2-\rho^{2}\right)-\sigma \rho-1\right)\left(\psi\left(4-\rho^{2}\right)-\sigma \rho-2\right)},
$$

which immediately implies $\dot{q}_{1}^{*}<\dot{q}_{1}^{Q}$ by A1. Hence $q_{1}^{Q}(\theta) \leq q_{1}^{*}(\theta)$ for all $\theta$ with equality only at $\bar{\theta}$.

Hence, we get $\dot{e}_{1}^{Q}=\dot{q}_{1}^{Q} / \psi>\dot{q}_{1}^{*} / \psi=\dot{e}_{1}^{*}$. Since $e_{1}^{Q}(\bar{\theta})=e_{1}^{*}(\bar{\theta})$, this implies $e_{1}^{Q}(\theta) \leq e_{1}^{*}(\theta)$ for all $\theta \leq \bar{\theta}$.

Using the expressions above of $\dot{q}_{1}^{Q}$ and $\dot{e}_{1}^{Q}$, we obtain:

$$
1+\rho \dot{q}_{2}^{Q}=\frac{(2 \psi-1)(2+\rho)}{2\left(\psi\left(2-\rho^{2}\right)-\sigma \rho-1\right)}>0
$$

when A1 holds.

Program $\left(\mathcal{P}^{Q \prime}\right)$ displays positive interior solutions whenever $\underline{\theta}$ is high enough. Hence, for $q_{1}^{Q}(\theta)$ being positive, (21) implies:

$$
0<\dot{U}_{1}^{Q}(\theta)=\frac{q_{1}^{Q}(\theta)(2 \psi-1)(2+\rho)}{2\left(\psi\left(2-\rho^{2}\right)-\sigma \rho-1\right)}
$$

so that $U_{1}^{Q}(\cdot)$ is increasing in $\theta$. 
Together with $\dot{q}_{1}^{Q}>0,(21)$ also implies that the second-order conditions of the retailer $R_{1}$ 's problem, namely (13), hold since

$$
\dot{q}_{1}^{Q}(\theta)\left(1+\rho \dot{q}_{2}^{Q}(\theta)\right)=\dot{q}_{1}^{Q}\left(1+\rho \dot{q}_{2}^{Q}\right)>0 .
$$

Finally, let $U_{1}^{Q}(\theta, \hat{\theta})$ define the retailer's profits when the true demand realization is $\theta$ but he announces a message $\hat{\theta} \neq \theta$ to $S_{1}$. To show that global incentive compatibility constraints hold we must have $\Gamma^{Q}(\theta, \hat{\theta}) \equiv U_{1}^{Q}(\theta, \theta)-U_{1}^{Q}(\theta, \hat{\theta}) \geq 0$ for each pair $(\theta, \hat{\theta}) \in \Theta^{2}$. Simple algebraic manipulations allow to rewrite $\Gamma^{Q}(\cdot)$ as:

$$
\Gamma^{Q}(\theta, \hat{\theta})=\int_{\hat{\theta}}^{\theta}\left\{-\dot{t}_{1}^{Q}(s)+\dot{q}_{1}^{Q}(s)\left(p_{1}\left(\theta, e_{1}^{Q}(s), q_{1}^{Q}(s), q_{2}^{Q}(\theta)\right)-q_{1}^{Q}(s)\right)\right\} d s .
$$

Using (12), we get $\dot{t}^{Q}(s) \equiv \dot{q}_{1}^{Q}(s)\left(p_{1}\left(s, e_{1}^{Q}(s), q_{1}^{Q}(s), q_{2}^{Q}(s)\right)-q_{1}^{Q}(s)\right)$. Inserting into the above equation, we get:

$$
\Gamma^{Q}(\theta, \hat{\theta})=\int_{\hat{\theta}}^{\theta} \dot{q}_{1}^{Q}(s)\left\{\int_{s}^{\theta}\left(1+\rho \dot{q}_{2}^{Q}(x)\right) d x\right\} d s=\dot{q}_{1}^{Q}\left(1+\rho \dot{q}_{2}^{Q}\right) \frac{(\theta-\hat{\theta})^{2}}{2} \geq 0 \quad \forall(\theta, \hat{\theta})
$$

where the last equality follows from the fact that the equilibrium is linear in types and the final inequality follows from (22) which concludes the proof.

\subsection{Proof of Proposition 1}

Because of linearity and the fact that $e_{1}^{Q}(\bar{\theta})=e_{1}^{P}(\bar{\theta})$, we have $e_{1}^{Q}(\theta)-e_{1}^{P}(\theta)=\left(\dot{e}_{1}^{P}-\right.$ $\left.\dot{e}_{1}^{Q}\right)(\bar{\theta}-\theta)$ for all $\theta$. Using the definition of $\dot{e}_{1}^{P}$ and $\dot{e}_{1}^{Q}, \mathbf{A} \mathbf{1}$ implies:

$$
\dot{e}_{1}^{P}-\dot{e}_{1}^{Q}=\frac{(2 \psi-1)(2+\rho)\left(\psi\left(2-\rho^{2}\right)-1\right)}{(2 \psi(2-\rho(\sigma+\rho))-\sigma \rho-2)\left(\psi\left(2-\rho^{2}\right)-\sigma \rho-1\right)}>0 .
$$

Since $e^{Q}(\bar{\theta})=e^{P}(\bar{\theta})$, we have $e^{Q}(\theta) \geq e^{P}(\theta)$ for all $\theta$ with equality only at $\bar{\theta}$.

We have also $q_{2}^{Q}(\theta)-q_{2}^{P}(\theta)=\left(\dot{q}_{2}^{P}-\dot{q}_{2}^{Q}\right)(\bar{\theta}-\theta)$ for all $\theta$, where:

$$
\dot{q}_{2}^{P}-\dot{q}_{2}^{Q}=\frac{\sigma(2+\rho)(2 \psi-1)^{2}}{2(2 \psi(2-\rho(\sigma+\rho))-\sigma \rho-2)\left(\psi\left(2-\rho^{2}\right)-\sigma \rho-1\right)} .
$$

Since $q_{2}^{Q}(\bar{\theta})=q_{2}^{P}(\bar{\theta})$, it follows that $\operatorname{sign}\left(q_{2}^{Q}(\theta)-q_{2}^{P}(\theta)\right)=\operatorname{sign}(\sigma)$ when A1 holds.

Similarly, we have:

$$
\dot{q}_{1}^{P}-\dot{q}_{1}^{Q}=\frac{\sigma \rho(2 \psi-1)(2+\rho) \psi}{(2 \psi(2-\rho(\sigma+\rho))-\sigma \rho-2)\left(\psi\left(2-\rho^{2}\right)-\sigma \rho-1\right)} .
$$

Finally, the facts that $q_{1}^{Q}(\bar{\theta})=q_{1}^{P}(\bar{\theta})$ and that $\mathbf{A} \mathbf{1}$ holds ensure that $\operatorname{sign}\left(q_{1}^{Q}(\theta)-\right.$ $\left.q_{1}^{P}(\theta)\right)=\operatorname{sign}(\sigma \rho)$. 


\subsection{Proof of Theorem 1}

First, observe that with a uniform distribution one has $\mathrm{E}_{\theta}[\bar{\theta}-\theta]=\Delta \theta / 2$ and $\mathrm{E}_{\theta}[\bar{\theta}-\theta]^{2}=$ $\Delta \theta^{2} / 3$. Second, since the disutility function is quadratic, inverse demand is linear, and equilibrium outputs and effort are linear, $S_{1}$ 's expected profit under both contractual regimes will be quadratic in $\theta$ and can be expressed as:

$$
\Pi_{1}^{\omega}=\mathrm{E}_{\theta}\left[\Pi_{1}^{\omega}(\theta)\right]=\Pi_{1}^{\omega}(\bar{\theta})-\dot{\Pi}_{1}^{\omega}(\bar{\theta}) \mathrm{E}_{\theta}[\bar{\theta}-\theta]+\frac{1}{2} \ddot{\Pi}_{1}^{\omega}(\bar{\theta}) \mathrm{E}_{\theta}[\bar{\theta}-\theta]^{2} \text { for each } \omega \in\{Q, P\}
$$

where $\Pi_{1}^{\omega}(\theta)$ is the type-contingent virtual profit in state $\theta$ when the optimal contract is chosen in class $\omega$. We have:

$$
\Pi_{1}^{P}(\theta)=\left(\theta+e_{1}^{P}(\theta)+\rho q_{2}^{P}(\theta)-q_{1}^{P}(\theta)\right) q_{1}^{P}(\theta)-\psi \frac{\left(e_{1}^{P}(\theta)\right)^{2}}{2}-(\bar{\theta}-\theta)\left(1+\rho \dot{q}_{2}^{P}\right) \psi e_{1}^{P}(\theta)
$$

and

$$
\Pi_{1}^{Q}(\theta)=\left(\theta+e_{1}^{Q}(\theta)+\rho q_{2}^{Q}(\theta)-q_{1}^{Q}(\theta)\right) q_{1}^{Q}(\theta)-\psi \frac{\left(e_{1}^{Q}(\theta)\right)^{2}}{2}-(\bar{\theta}-\theta)\left(1+\rho \dot{q}_{2}^{Q}\right) q_{1}^{Q}(\theta)
$$

with $\psi e_{1}^{Q}(\theta)=q_{1}^{Q}(\theta)$.

Using (24) and taking expectations, we get:

$$
\Pi_{1}^{Q}-\Pi_{1}^{P}=\left(\dot{\Pi}_{1}^{P}(\bar{\theta})-\dot{\Pi}_{1}^{Q}(\bar{\theta})\right) \frac{\Delta \theta}{2}+\left(\ddot{\Pi}_{1}^{Q}(\bar{\theta})-\ddot{\Pi}_{1}^{P}(\bar{\theta})\right) \frac{\Delta \theta^{2}}{6},
$$

We need then to compute each term appearing in (25). First, consider program $\left(\mathcal{P}^{P \prime}\right)$, differentiating with respect to $\theta$ the maximand and using the Envelope Theorem, we have:

$$
\dot{\Pi}_{1}^{P}(\theta)=\left(1+\rho \dot{q}_{2}^{P}\right)\left(\psi e_{1}^{P}(\theta)+q_{1}^{P}(\theta)\right)
$$

and thus, since $\psi e_{1}^{P}(\bar{\theta})=q_{1}^{P}(\bar{\theta})$, we get $\dot{\Pi}_{1}^{P}(\bar{\theta})=2 q_{1}^{*}(\bar{\theta})\left(1+\rho \dot{q}_{2}^{P}\right)$. Differentiating once more the expression of $\dot{\Pi}_{1}^{P}(\theta)$ above w.r.t. $\theta$ and taking into account the linearity of $e_{1}^{P}(\theta)$ and $q_{1}^{P}(\theta)$, we get $\ddot{\Pi}_{1}^{P}(\bar{\theta})=\left(1+\rho \dot{q}_{2}^{P}\right)\left(\dot{q}_{1}^{P}+\psi \dot{e}_{1}^{P}\right)$.

Consider now program $\left(\mathcal{P}^{Q^{\prime}}\right)$, differentiating with respect to $\theta$ the maximand and using the Envelope Theorem, we have:

$$
\dot{\Pi}_{1}^{Q}(\theta)=2\left(1+\rho \dot{q}_{2}^{Q}\right) q_{1}^{Q}(\theta) .
$$

Therefore, we get $\dot{\Pi}_{1}^{Q}(\bar{\theta})=2\left(1+\rho \dot{q}_{2}^{Q}\right) q_{1}^{*}(\bar{\theta})$. Differentiating once more w.r.t. $\theta$ and taking into account the linearity of $q_{1}^{Q}(\theta)$, we get $\ddot{\Pi}_{1}^{Q}(\bar{\theta})=2 \dot{q}_{1}^{Q}\left(1+\rho \dot{q}_{2}^{Q}\right)=\left(\dot{q}_{1}^{Q}+\psi \dot{e}_{1}^{Q}\right)\left(1+\rho \dot{q}_{2}^{Q}\right)$.

Substituting $\dot{\Pi}_{1}^{\omega}(\bar{\theta})$ and $\ddot{\Pi}_{1}^{\omega}(\bar{\theta})$, for $\omega \in\{Q, P\}$, into (25) yields:

$$
\Pi_{1}^{Q}-\Pi_{1}^{P}=\rho q_{1}^{*}(\bar{\theta})\left(\dot{q}_{2}^{P}-\dot{q}_{2}^{Q}\right) \Delta \theta-\left(\left(\dot{q}_{1}^{P}+\psi \dot{e}_{1}^{P}\right)\left(1+\rho \dot{q}_{2}^{P}\right)-\left(\dot{q}_{1}^{Q}+\psi \dot{e}_{1}^{Q}\right)\left(1+\rho \dot{q}_{2}^{Q}\right)\right) \frac{\Delta \theta^{2}}{6} .
$$

Taking $\Delta \theta$ small enough and substituting (23) into the above equation we have up to terms of order $\Delta \theta^{2}$ when $\rho \sigma>0$ :

$$
\Pi_{1}^{Q}-\Pi_{1}^{P} \approx \frac{\sigma \rho q_{1}^{*}(\bar{\theta})(2 \psi-1)(2+\rho) \psi \Delta \theta}{(2 \psi(2-\rho(\sigma+\rho))-\sigma \rho-2)\left(\psi\left(2-\rho^{2}\right)-\sigma \rho-1\right)},
$$


which immediately yields the result since $\Pi_{1}^{Q}-\Pi_{1}^{P}>0$ whenever $\rho \sigma>0$, and $\Pi_{1}^{Q}-\Pi_{1}^{P}<0$ whenever $\rho \sigma<0$.

To conclude the proof we must consider the case where $\sigma \rho=0$. The first-order term is now not enough to sign $\Pi_{1}^{Q}-\Pi_{1}^{P}$, hence we will use the second-order term of (25):

$$
\Pi_{1}^{Q}-\Pi_{1}^{P}=\left(\left(\dot{q}_{1}^{Q}+\psi \dot{e}_{1}^{Q}\right)\left(1+\rho \dot{q}_{2}^{Q}\right)-\left(\dot{q}_{1}^{P}+\psi \dot{e}_{1}^{P}\right)\left(1+\rho \dot{q}_{2}^{P}\right)\right) \frac{\Delta \theta^{2}}{6} .
$$

First assume $\sigma=0$, in this case $\dot{q}_{1}^{P}=\dot{q}_{1}^{Q}$ and $\dot{q}_{2}^{P}=\dot{q}_{2}^{Q}$, and we have:

$$
\Pi_{1}^{Q}-\Pi_{1}^{P}=-\psi\left(1+\rho \dot{q}_{2}^{Q}\right)\left(\dot{e}_{1}^{P}-\dot{e}_{1}^{Q}\right) \frac{\Delta \theta^{2}}{6}
$$

which yields the result since $\dot{e}_{1}^{P}>\dot{e}_{1}^{Q}$. The same kind of arguments shows that, when $\rho=0$,

$$
\Pi_{1}^{Q}-\Pi_{1}^{P}=-\psi\left(\dot{e}_{1}^{P}-\dot{e}_{1}^{Q}\right) \frac{\Delta \theta^{2}}{6}<0
$$

which concludes the proof.

\section{References}

[1] Aghion, P., and Hermalin, B., (1990), "Legal Restrictions on Private Contracts Can Enhance Efficiency" The Journal of Law, Economics and Organization, 6: 381-409

[2] Allen, F., and Gale, D., (1992), "Measurement Distortion and Missing Contingencies Optimal Contracts", Economic Theory, 2: 1-26.

[3] Bernheim, B. D., and Whinston M. D., (1998), "Incomplete Contracts and Strategic Ambiguity", American Economic Review, 88: 902-932.

[4] Blair, F. B., and Lewis, T.R., (1994), "Optimal Retail Contracts with Asymmetric Information and Moral Hazard", Rand Journal of Economics, 25: 284-296.

[5] Bollard, A. E., (1989), "An Economic Comment on the Commerce Act Review", mimeo.

[6] Bork, R., (1978), The Antitrust Paradox, Free Press, New-York.

[7] Caillaud, B., Jullien, B. and Picard, P., (1995), "Competing Vertical Structures: Precommitment and Renegotiation", Econometrica, 63: 621-646.

[8] Caillaud, B., and Rey, P., (1995), "Strategic Ignorance in Producer-Distributor Relationship", mimeo University of Toulouse.

[9] Chevalier, J. A., Kashyap, J. A. and Rossi, P. A., (2003), "Why Don't Prices Rise during Periods of Peak Demand? Evidence from Scanner Data", American Economic Review, 93: 15-37.

[10] Crémer, J., (1995), "Arm's Length Relationships", Quarterly Journal of Economics, 110: 275-295. 
[11] Dessi, R., (2007), "Contractual Enforcement and Strategic Incompleteness" IDEI Working Paper, n. 465.

[12] Dewatripont, M., and Maskin, E., (1995), "Contractual Contingencies and Renegotiation", Rand Journal of Economics, 26: 704-719.

[13] Dewatripont, M., and Maskin, E., (1990), "Contract Renegotiation in Models of Asymmetric Information", European Economic Review, 34: 311-321.

[14] Fershtman, C., and Judd, K., (1987), "Equilibrium Incentives in Oligopoly", American Economic Review, 77: 551-559.

[15] Fershtman, C., Judd, K., and E. Kalai, (1991), "Observable Contracts, Strategic Delegation and Cooperation", International Economic Review, 32: 551-559.

[16] Gal-Or, E., (1991a), "A Common Agency with Incomplete Information", Rand Journal of Economics, 22: 274-286.

[17] Gal-Or, E., (1991b), "Duopolistic Vertical Restraints", European Economic Review, 35: $1237-1253$.

[18] Gal-Or, E., (1991c), "Vertical Restraints with Incomplete Information", The Journal of Industrial Economics, 39: 503-516.

[19] Gal-Or, E., (1991d), "Vertical Integration in Oligopoly", The Journal of Law and Economic Organization, 8: 377-393.

[20] Gal-Or, E., (1999), "Vertical Integration or Separation of the Sales Functions as Implied by Competitive Forces", International Journal of Industrial Organization, 17: 641-662.

[21] Kastl J., Martimort, D. and Piccolo, S., (2008) "Resale Price Maintenance under Asymmetric Information: Competing Hierarchies and Retail Externalities," mimeo.

[22] Katz, M., (1991), "Game-Playing Agents: Unobservable Contracts as Precommitments", Rand Journal of Economics, 22: 1-31.

[23] Kuhn, K.U., (1997), "Nonlinear Pricing in Vertically Related Duopolies", Rand Journal of Economics, 28: 37-62.

[24] Laffont, J.J., and Martimort, D., (2002), The Theory of Incentives: The PrincipalAgent Model, Princeton University Press.

[25] Laffont, J.J., and Tirole, J., (1986), "Using Cost Observation to Regulate Firms", Journal of Political Economy, 94: 614-641.

[26] Laffont, J.J., and Tirole, J., (1993), A Theory of Incentives in Procurement and Regulation, MIT Press, Cambridge.

[27] Lafontaine, F., and Slade, M., (1997), "Retail Contracting: Theory and Practice", The Journal of Industrial Economics, 45: 1-25. 
[28] Martimort, D., (1996), "Exclusive Dealing, Common Agency, and Multiprincipals Incentive Theory", Rand Journal of Economics, 27: 1-31.

[29] Martimort, D., (1999), "Renegotiation Design with Multiple Regulators", Journal of Economic Theory, 88: 261-293.

[30] Martimort D., and Piccolo, S., (2007), "Resale Price Maintenance under Asymmetric Information", International Journal of Industrial Organization, 25: 315-339.

[31] Martimort D., and Piccolo, S., (2008), "The Strategic Value of Quantity Forcing Contracts", CSEF working paper \# 160.

[32] Mathewson, G., and Winter R., (1984), "An Economic Theory of Vertical Restraints", Rand Journal of Economics, 15: 27-38.

[33] Myerson, R., (1982), "Optimal Coordination Mechanisms in Generalized PrincipalAgent Problems", Journal of Mathematical Economics, 10: 67-81.

[34] Olsen, T., and Torsvik, G., (1993), "The Ratchet Effect in Common Agency: Implications for Regulation and Privatization" The Journal of Law, Economics and Organization, 9: 136-158.

[35] Pearce, D., and Stachetti, E., (1998), "The Interaction of Implicit and Explicit Contracts in Repeated Agency", Games and Economic Behavior, 23: 75-96.

[36] Piccolo S., D'Amato, M. and Martina, R., (2008), "Product Market Competition and Organizational Slack under Profit-Target Contracts", International Journal of Industrial Organization, 26: 1389-1406.

[37] Rey, P., and Stiglitz, J., (1995), "The Role of Exclusive Territories in Producers' Competition", Rand Journal of Economics, 26: 432-451.

[38] Rey, P., and Tirole, J., (1986), "The Logic of Vertical Restraints", American Economic Review, 76: 921-939.

[39] Schmidt, K., and Schnitzer, M., (1995), "The Interaction of Explicit and Implicit Contracts", Economic Letters, 48: 193-199.

[40] Segal, I., (1999), "Complexity and Renegotiation: A Foundation for Incomplete Contracts," Review of Economic Studies, 66: 57-82.

[41] Sheppard, A., (1993), "Contractual Form, Retail Price, and Asset Characteristics in Gasoline Retailing", Rand Journal of Economics, 24: 58-77.

[42] Sklivas, S., (1987), "The Strategic Choice of Managerial Incentives" RAND Journal of Economics, 18: 452-458.

[43] Spier, K., (1992), "Incomplete Contracts in a Model with Adverse Selection and Exogenous Costs of Enforcement", Rand Journal of Economics, 23: 432-443.

[44] Tirole, J., (1999), "Incomplete Contracts: Where Do We Stand?, Econometrica, 67: 741-781. 